\title{
Use of a Website to Accomplish Health Behavior Change: If You Build It, Will They Come? And Will It Work If They Do?
}

\author{
W. Perry Dickinson, MD, Russell E. Glasgow, PhD, Lawrence Fisher, PhD, \\ L. Miriam Dickinson, PhD, Steven M. Christensen, Paul A. Estabrooks, PhD, \\ and Benjamin F. Miller, PsyD
}

Purpose: This article describes the development, implementation, and effectiveness of 2 interactive websites designed to support health behavior change around healthy eating, physical activity, smoking, and use of alcohol for primary care patients.

Methods: Patients from 6 primary care practices were recruited and randomized to a basic website (including a health assessment with feedback of the results and educational materials about health behavior change) or an enhanced website that included the features of the basic site plus an action planning component. Patients were prompted to return for follow-up assessments at 3 and 6 months after enrollment.

Results: Of 7706 participants, $169(2.2 \%)$ targeted for recruitment actually used the website. Both web-based interventions seemed to assist patients with making positive changes in their behavior, especially activity level and healthful diet. There were no significant differences in the effectiveness of the basic and enhanced websites.

Conclusions: Interactive behavior-change technology interventions can assist primary care patients and practices in health behavior change activities. Difficulties with patient recruitment and the lack of added effectiveness of the enhanced website suggest that such interventions work better if integrated into the interaction between primary care clinicians and patients rather than as a standalone intervention. (J Am Board Fam Med 2013;26:168-176.)

Keywords: Behavior Modification, Health Care Systems, Health Services, Lifestyle, Practice-based Research, Primary Health Care

Increasing emphasis in primary care is being placed on the cost-efficient delivery of high-quality primary and secondary preventive care using evidence-based

This article was externally peer reviewed.

Submitted 29 December 2011; revised 4 October 2012; accepted 23 October 2012.

From the Department of Family Medicine, University of Colorado Denver, Aurora (WPD, LMD, BFM); the Division of Cancer Control and Population Sciences, National Cancer Institute (REG); the Diabetes Center, University of California, San Francisco (LF); InterVision Media, Eugene, OR (SMC); and the Department of Human Nutrition, Foods and Exercise, Virginia Tech (PAE).

Funding: The Robert Wood Johnson Foundation provided the funding for this project through its Prescription for Health initiative.

Conflict of interest: none declared.

Corresponding author: Perry Dickinson, University of Colorado Denver, Department of Family Medicine, Mail Stop F496, Academic Office 1, 12631 East 17th Avenue, Room 3223, Aurora, CO 80045 (E-mail: perry.dickinson@ucdenver.edu). interventions, in part because of the growing implementation of the Chronic Care Model and the patient-centered medical home. ${ }^{1-7}$ The U.S. Preventive Services Task Force (USPSTF) has identified and strongly recommends theory-based behavioral strategies that can lead to significant behavior change. ${ }^{8-10}$ However, many of the approaches recommended by the USPSTF suffer from suboptimal implementation in real-world settings. A major challenge at this point is to package and deliver health behavior change interventions in ways that are practical, consistent, time-efficient for physicians and staff, and can reach and engage a high percentage of patients and practices." ${ }^{11}$ Primary care physicians are overwhelmed by competing clinical demands, and preventive care and health behavior change often are left unaddressed. ${ }^{12}$ 
Stange et $\mathrm{al}^{13}$ concluded that 1 minute is the average amount of time that primary care physicians can devote to prevention during a typical office visit. Yarnall et al ${ }^{14}$ determined that 7.4 hours per day would be required for a primary care physician to deliver recommended preventive care services to an average patient panel. In an overview of interactive behavior change technology (IBCT), Glasgow et $\mathrm{al}^{15}$ stated that since it is impossible for primary care physicians to provide all recommended preventive care personally, IBCT may be essential to achieving comprehensive and consistent delivery of health behavior counseling. They concluded that, "if constructed to draw on the strengths of primary care and to use patient-centered principles, IBCT can inform, leverage, and support patient-provider communication and enhance behavior change."

A large body of research highlights the importance of individual autonomy and patient choice in successful behavior change, ${ }^{16-19}$ which is the basis of patient-centered medicine. ${ }^{20}$ When patients are given a choice of health behaviors to address, they elect to change behavior in areas of most perceived personal need, and they are more likely to change the targeted health behavior successfully. ${ }^{21} \mathrm{We}$ can increase patient engagement and the likelihood of successful behavior change by giving patients choices regarding (1) which targeted behaviors to address, (2) the pace and timing of behavioral change, and (3) which strategies to use to accomplish change.

The key processes for health behavior counseling are summarized by the 5 As approach advocated by the USPSTF and others..$^{10,22,23}$ Because the time, expertise, and resources required to address this behavioral process usually are not available during clinical encounters, in this study we attempted to automate the application of the 5 As using interactive technologies through the Internet. $^{24}$

The goal of this project was to develop, implement, and assess a web-based program for health behavior change for primary care based on the $5 \mathrm{As}$ and using patient-centered approaches and costand time-efficient interactive technologies. Two closely related websites were developed to determine (1) the benefit of a site providing a health risk appraisal with patient feedback followed by the provision of multiple resources supporting behavior change and (2) the added benefit of online action planning modules to further assist patients in planning and implementing health behavior change activities. The purposes of this article are to describe the development and implementation of the Connection to Health (CTH) interactive programs, the results and lessons learned from recruiting for and implementing CTH, and the implications for future attempts to use IBCT to enhance preventive care and health risk reduction in primary care.

\section{Methods}

\section{Website Development}

The CTH websites were developed based on previous projects using somewhat similar web-based interventions for health behavior change in both general populations and patients with diabetes. ${ }^{24-27}$ Details of the 2 websites are included below. Our website was initially piloted in an interactive manner with a 12-person patient advisory group and through an interactive, individual review of the site with 4 clinicians who were not a part of the study; changes were made based on the feedback from those groups.

\section{Practice Recruitment}

A diverse set of 6 practices were recruited from 2 practice-based research networks (PBRNs): the Colorado Research Network (CaReNet), serving underserved populations, and the High Plains Research Network (HPRN), based in the rural Plains region of northeast Colorado. Four practices (2 urban residency practices, an urban community health center, and an urban private practice) enrolled after a presentation regarding the study at a yearly CaReNet convocation. Two rural HPRN practices enrolled after being approached directly to diversify the study practices.

\section{Patient Recruitment}

Patients from each practice were eligible to participate if they were between the ages of 18 and 65, had been seen in the practice within the last 18 months, and could read and write English or Spanish. All website and written materials were available in both English and Spanish. Our institutional review board required that potential subjects be given a paper copy of both the informed consent form and the Health Insurance Portability and Accountability Act (HIPAA) waiver before obtaining formal 
patient consent over the Internet. Therefore, we used 2 patient recruitment strategies. Practices generated a list of patients seen over the past 18 months. Our research staff mailed a letter from their doctor's office to a randomly selected group of up to 1500 patients from each practice, inviting them to participate in the project; this letter was accompanied by the paper copies of the consent and HIPAA waiver. In addition, the clinicians in each practice were given the option of distributing recruitment packets containing the consent form and HIPAA waiver directly to patients. Using the first strategy, we mailed 7646 recruitment letters to patients in the 6 practices. Based on the number of packets given to and used at the various practices, we estimated that approximately 150 recruitment packets were distributed by the clinicians. Some of the 150 patients receiving packets from their clinicians also would have received the mailing, but the exact extent of the overlap is unknown. Estimating the overlap at $60 \%$ would result in an additional 60 patients receiving recruitment materials, for a total of 7706 patients receiving recruitment materials. Of that number, 169 patients $(2.2 \%$ of those invited) enrolled in the project.

\section{Design and Flow of the Connection to Health Web-based Intervention}

Interested patients were asked to go to the website and enroll in the project. Upon initial arrival at the website, patients were prompted to complete the online consent and HIPAA waiver. They then were asked to complete a health risk appraisal that included the measures described below. $\mathrm{Pa}$ tients were given feedback on the results, with recommendations regarding health behaviors that they might wish to consider modifying. Patients then were randomized to either the basic or enhanced CTH websites. The basic site included extensive educational materials regarding 4 health behaviors (healthful eating, activity level, alcohol intake, and cigarette smoking) and depression, including materials designed to assist patients in behavior change. The basic site also included regularly updated tips regarding behavior change and an educational section dealing with how they could better communicate with their clinicians about behavior change and related issues. The enhanced site included all the elements of the basic site plus an extensive section about action plans, where patients were prompted to develop an individualized action plan for changing any of the 4 targeted behaviors (activity level, eating behaviors, smoking, or alcohol use) or a plan to monitor their depression symptoms. Patients who developed an action plan were prompted to print out the action plan for their own use and to discuss with their primary care clinician at their next office visit. The enhanced site also included a discussion forum section, where patients could post issues and discuss them with other patients working on similar behavioral changes, and an "Ask the Expert" section, where patients could pose questions for the clinical team. Patients were encouraged to return to the site periodically; those completing action plans were prompted regularly by E-mails or automated phone calls to reassess their progress and modify their action plans. All patients were encouraged by Emails and automated phone calls to return to the site 3 and 6 months after enrollment to reassess their health behaviors.

\section{Measures}

For the Prescription for Health Program, a common set of behavioral measures derived from existing behavioral scales was required across all projects, as detailed in previous publications. ${ }^{28,29}$ These measures included the following:

1. Diet: Derived from Ammerman's "Starting the Conversation" research, the 7-item diet instrument asked about intake of fast food, fruits/ vegetables, sweet drinks, protein, chips/crackers, desserts, and fats, with ordinal values for each response representing the amount consumed. ${ }^{30,31}$ Summing the values provided a score of 0 to 14 , where 14 represented the least healthy diet habits.

2. Physical activity: The International Physical Activity Questionnaire short form is a 7-item scale that reports minutes per week of light, moderate, and strenuous physical activity. ${ }^{32}$ For these analyses, underactive was defined as $<150$ minutes per week of moderate exercise, vigorous exercise, or both.

3. Smoking: The 4 smoking items were derived from 3 national smoking surveys. ${ }^{33-35}$ Current smokers were defined as smoking at least 100 cigarettes in their lifetime and having smoked within the past 30 days.

4. Alcohol: Three questions from the Behavioral Risk Factor Surveillance System 2003 were 
used to assess alcohol consumption in the past 30 days. ${ }^{35}$ Heavy drinking was defined as $>2$ drinks per day for men and $>1$ drink per day for women.

5. Risk factors: The total number of risk factors was calculated as the number of positive items from the dichotomized health behavior domains, including unhealthful diet, underactive, current smoker, and heavy drinker. ${ }^{36}$

6. In addition to the required behavioral measures, we screened for and monitored depressive symptoms using the Patient Health Questionnaire (PHQ), a self-administered version of the PRIME-MD diagnostic instrument for common mental disorders. ${ }^{37} \mathrm{We}$ also assessed health-related quality of life using the 4-item version of the Centers for Disease Control and Prevention Healthy Days measure. ${ }^{38}$

\section{Analysis}

Descriptive statistics were calculated for sociodemographic and clinical variables. $\chi^{2}$ Tests and $t$ tests were used to compare baseline patient characteristics between patients randomized to the enhanced versus basic intervention groups. Primary outcome measures were scores at baseline and 3 and 6 months for diet, physical activity, quality of life, number of health risk behaviors, and depression measures. General linear mixed effects models were used to compare change in scores on continuous or ordinal outcomes over time for patients in the 2 intervention groups. ${ }^{39}$ Generalized linear models were used for the binary outcome of physical activity (active vs underactive). Methods that utilized all available data were used for all analyses, assuming missing data that could be ignored. ${ }^{40,41}$ Repeated measures within patients were modeled with time and coded as a categorical variable. Sociodemographic covariates were included if they were significantly associated with the outcome, differed between the treatment groups, or were associated with dropout. All analyses were conducted with SAS version 9.2 (SAS Inc., Cary, NC).

\section{Results}

\section{Patient Characteristics}

Figure 1 shows details of patient enrollment, randomization, and follow-up. Eighty-eight patients were randomized to the enhanced site and 81 to the basic site. Patient sociodemographic and clinical characteristics, shown in Table 1 , were similar for

Figure 1. CONSORT diagram. *One patient withdrew from the study because they did not want to receive the reminder E-mails or telephone calls associated with prompting patients to return to the website to update their action plans for their behavior changes.

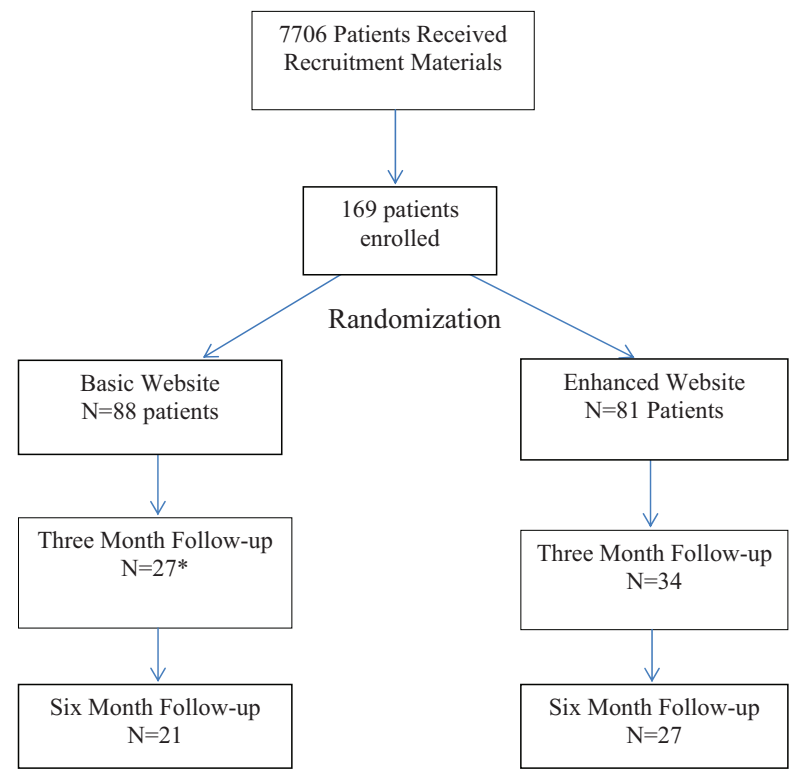

* One patient withdrew from the study due to not wanting to receive the reminder emails or telephone calls associated with prompting patients to return to the web site to update their action plans for their behavior changes. 
Table 1. Patient Characteristics at Baseline

\begin{tabular}{|c|c|c|c|}
\hline Variable & Basic Site $(\mathrm{n}=81)$ & Enhanced Site $(\mathrm{n}=88)$ & Total $(\mathrm{n}=169)$ \\
\hline Age, yrs & $42.5(13.6)$ & $43.4(13.1)$ & - \\
\hline Male sex $(\%)$ & 24.7 & 17.1 & 20.7 \\
\hline Non-Hispanic white (\%) & 88.9 & 82.9 & 85.8 \\
\hline Some college or college graduate (\%) & 92.6 & 84.09 & 88.2 \\
\hline \multicolumn{4}{|l|}{ Income, \$ (\%) } \\
\hline$<25,000$ & 17.3 & 12.5 & 14.8 \\
\hline $25,000-49,999$ & 22.2 & 26.1 & 24.3 \\
\hline$\geq 50,000$ & 60.5 & 61.4 & 60.9 \\
\hline BMI & $27.5(5.9)$ & $28.3(7.2)$ & $27.9(6.6)$ \\
\hline Physical health (unhealthy days)* & $3.0(4.7)$ & $5.0(7.8)$ & $4.1(6.6)$ \\
\hline Mental health (unhealthy days) & $6.0(7.7)$ & $5.0(7.1)$ & $5.5(7.4)$ \\
\hline History of depression (\%) & 40.1 & 34.1 & 37.3 \\
\hline Treated for current depression (\%) & 24.7 & 17.1 & 20.7 \\
\hline Mean PHQ score & $5.35(4.76)$ & $4.77(4.34)$ & $5.05(4.54)$ \\
\hline PHQ score $\geq 10(\%)$ & 16.0 & 13.6 & 14.8 \\
\hline Baseline depression $^{\dagger}$ & 32.1 & 25.0 & 28.4 \\
\hline Chronic medical conditions & $0.72(0.94)$ & $0.72(0.99)$ & $0.72(0.98)$ \\
\hline Current smoker* (\%) & 3.7 & 12.5 & 8.3 \\
\hline Underactive (\%) & 63.0 & 61.4 & 62.1 \\
\hline Diet score ${ }^{\ddagger}$ & $5.21(2.42)$ & $5.15(2.65)$ & $5.18(2.54)$ \\
\hline Heavy drinking (\%) & 13.6 & 10.2 & 11.8 \\
\hline Risk factors $(0-4)$ & $1.68(0.80)$ & $1.74(0.93)$ & $1.71(0.86)$ \\
\hline
\end{tabular}

Values provided as mean (SD) unless otherwise indicated.

${ }^{*} P<.05$.

${ }^{\dagger}$ Currently being treated, 9-item Patient Health Questionnaire (PHQ) score $\geq 10$, or both.

${ }^{\ddagger}$ Lower is better; range, $0-12$.

BMI, body mass index.

the 2 groups; the only significant differences at baseline were that there were more smokers in the enhanced intervention group $(P=.0118)$, and the patients in the enhanced group indicated that they had poorer physical health based on a higher number of unhealthy days during the previous month $(P=.0472)$. The mean age was 43 years (range, $18-79$ years), $79.7 \%$ were women, $85.8 \%$ were non-Hispanic white, $39 \%$ had income levels of $<\$ 50,000,88.2 \%$ had at least some college or were college graduates, and they tended to be in the overweight body mass index category. Thirteen patients listed themselves as Hispanic, but only one used the Spanish version of the website.

The level of depression in this sample was surprisingly high: $37.3 \%$ reported a history of depression, $20.7 \%$ currently are being treated for depression, and $14.8 \%$ have a current 8 -item PHQ-8 score of $\geq 10$ (indicative of at least a moderate depression.) Combining the patients currently being treated with those not being treated but with an 8-item PHQ score of $\geq 10,28.4 \%$ had current depression at baseline.

Table 2 details the number of patients from the enhanced group who completed action plans in the various areas. Patients from both intervention groups tended to return to the site (a mean of 3.43 times), with no difference between the 2 groups. All patients were prompted to return at 3 and 6 months after baseline to complete follow-up health risk appraisals. Sixty-one patients completed the 3-month

Table 2. Use of Action Plans

\begin{tabular}{lcc}
\hline Area of Concern $(\mathrm{n}=88)$ & $\begin{array}{c}\text { Positive } \\
\text { Screens }\end{array}$ & $\begin{array}{c}\text { Positive Screen } \\
\text { With Action Plan }\end{array}$ \\
\hline Physical activity & $54(61.4)$ & $19(35.2)$ \\
Diet & $79(89.8)$ & $30(38)$ \\
9-Item PHQ score $\geq 10$ & $12(13.6)$ & $3(25)$ \\
Alcohol & $9(10.2)$ & $2(22.2)$ \\
Smoking & $11(12.5)$ & $2(18.2)$ \\
\hline
\end{tabular}

Values provided as n (\%).

PHQ, Patient Health Questionnaire. 
follow-up health risk appraisal and 48 patients completed the 6-month follow-up health risk appraisal (Figure 1). Those who completed the 3- and 6-month follow-up appraisals may have been more engaged in the intervention, as evidenced by their having returned for a mean of 6.1 and 7.4 visits to the website, respectively (again with no significant difference between the 2 intervention groups.) Subjects who completed at least one follow-up were more likely to be obese $(P=.0089)$ and less likely to be current smokers at baseline $(P=.0282)$. There were no differences between completers and noncompleters at 3 or 6 months by intervention group, sex, race, chronic medical conditions, education, income, baseline physical activity level, baseline health status rating, baseline physical health, heavy alcohol use, current depression, or poor diet at baseline (all $P>.10$ ). Information on reasons for not returning to the website was not available.

To investigate the impact of the interventions, we analyzed change in the behavioral outcome measures, comparing baseline to 3-and 6-month follow-up scores. Healthful eating scores improved significantly overall, with mean scores of 5.15 at baseline, 3.98 at 3 months, and 3.96 at 6 months $(\mathrm{F}(2163)=15.87 ; P<.0001)$. Improvements among patients from the enhanced site versus the basic site approached but did not attain statistical significance $(\mathrm{F}(2163)=2.61 ; P=.0770)$. Physical activity levels also improved significantly overall $(\mathrm{F}(2103)=5.02 ; P=.0083)$, but there were no significant between-group effects $(\mathrm{F}(2103)=0.34$; $P=.7137)$. Among subjects with at least one follow-up, 56\% were underactive at baseline compared with $46 \%$ at the 3 -month follow-up and 39\% at the 6-month follow-up. There were too few subjects who were smokers or heavy alcohol consumers at baseline to detect change over time. The overall number of health risk factors declined significantly in both groups $(\mathrm{F}(2162)=8.37 ; P=$ $.0003)$, with a reduction from 1.71 at baseline to 1.36 at 3 months and 1.46 at 6 months, but no between-group differences were found $(\mathrm{F}(2162)=$ 0.07; $P=$.9364). Similarly, 8-item PHQ scores improved over time in both groups $(\mathrm{F}(2163)=$ 5.24; $P=.0062)$, with no differences between groups $(\mathrm{F}(2163)=0.04 ; P=.9617)$. There were no significant effects on quality of life.

We analyzed whether indicators of patient engagement with or use of the site were related to change over time. There were no significant effects of having prepared an action plan or the number of visits to the website on any of the outcomes (all $P>$ .20). In addition, depression symptom scores at baseline were associated with worse diet scores $(P=.0089)$ but did not affect change in the diet scores. There was no effect of depression symptom scores on activity level or the overall number of risk factors. Finally, the patterns of patient usage of different portions of the site were reviewed. Beyond the core features of the site, including the assessment, feedback report, and action planning sections (for those in the enhanced site group), patients seemed to access and use frequently the sections with additional educational resources, whereas the discussion forum and "Ask the Experts" sections were seldom accessed or used.

\section{Discussion}

Both CTH web-based health behavior change interventions seem to assist patients with making positive changes in their behaviors, especially activity level and healthful diet. The relatively lower number of patients with problem drinking or smoking limited our ability to detect a change in those areas, and from the action plan data it seemed that patients were less likely to engage in trying to change those behaviors. These findings support the hypothesis that IBCT interventions can assist primary care patients and practices in health behavior change activities.

The lack of a significant difference in outcomes between the basic and the enhanced intervention sites warrants discussion. These findings could be very helpful in learning more about the correct amount and type of features related to increased website exposure. ${ }^{42}$ Other projects certainly suggest that the type of action planning process used in the enhanced CTH site should improve the user's ability to accomplish behavioral change. ${ }^{25-27,43}$ The elements shared on the basic and enhanced sites included the initial health risk appraisal with feedback and suggestions regarding desirable changes, extensive educational materials about the various health behaviors with suggestions regarding how to accomplish change, and regular follow-up with prompts to reassess their behaviors. The action planning portion of the enhanced website did not seem to increase the changes beyond those made by patients using the basic site. This does not 
mean that action planning is not important; rather, action planning done via a website without live reinforcement by a clinician was not incrementally effective in this study. Possible explanations for this finding include (1) filling out the health risk appraisal, getting feedback regarding needed change, and using a library of high-quality educational materials is sufficient to facilitate change, with no added impact from action planning; (2) the methodology used for the action planning in the enhanced website was inadequate and therefore did not have an impact (although the methodology had been successful in accomplishing change in other studies); (3) delivered in this fashion through a web-based intervention, action planning does not have additional impact, but if combined with more interaction with clinicians or others, we might see improved outcomes. This is suggested by the fact that prior studies using this action planning approach have included more human interaction than our current project. ${ }^{25,27,44,45}$ The amount of human interaction required to improve the effectiveness of this type of interactive behavior change technology intervention is an important area for future inquiry.

Patient recruitment was disappointing, with relatively low enrollment rates (169 of the 7706 who received recruitment materials enrolled). While practices and patients consistently expressed enthusiasm for the website in both piloting with a patient advisory group and in discussions with clinicians, patients largely did not proactively go to the site and enroll. It is unclear whether the extensive consent and HIPAA waiver procedure that patients had to complete online before getting to the real material of the site might have served as a deterrent to enrollment; since that was the first thing patients saw when they went to the site, it could have discouraged people from going further. In addition, the recruitment process allowed by the institutional review board was very passive. Practices and clinicians were allowed to give recruitment packets (which had to include a printed version of the consent and HIPAA waiver) directly to patients, but the bulk of the recruitment involved mailings to patients from the practices. It is also possible that the poor recruitment was due to a general lack of interest by patients in a standalone web tool of this type, despite the initial feedback. The potential added benefit for patient engagement with in-person interaction with clinicians-encouraging peo- ple to go to the site, engaging them in the behavior change process as facilitated by the site, and reinforcing the ongoing changes-were not part of this project but is a major issue for future studies of this type of IBCT intervention. This seems to be a repeated lesson learned from other related projects and deserves attention. However, a clear finding of this project is that if you build it, they may not come. We find that interactive technology can be a great tool, but it increasingly requires human interaction to effectively start and maintain the process.

The overall lack of use of the Spanish version of the site, after a costly effort to translate all website and recruitment materials into Spanish, is difficult to interpret. Do Spanish speaking patients generally not use web-based tools? Did the consent and HIPAA waiver materials particularly deter Spanishspeaking patients? We do not know the answers to these questions, but this is an important subset of the "if you build it, they may not come" lesson.

Limitations of this study included the low recruitment rate and relatively poor rate of return for 3- and 6-month follow-up surveys, which resulted in a sample that was not representative of the general populations of these practices. This could have resulted in a study cohort that was more motivated to change their behavior, influencing the results. This also limited our power in comparing the basic and enhanced study populations.

\section{Conclusions}

Health and behavior change is a complicated proposition made increasingly more difficult by the time demands placed on primary care providers. Despite the critical importance of and recent attention given to health and behavior change, there are no easy answers on how to address this problem. The assessment of health behaviors can be done through a web-based tool such as the one included in the CTH website. The completion of such a health behavior assessment, followed by feedback to the patient regarding the results and recommendation for changes and supplemented by the availability of online educational resources regarding health behaviors and change strategies, can result in changes in the health behaviors for patients. Health information technology interventions such as the one tested in this project can provide helpful tools for practices and patients, but to reach the most pa- 
tients they have to be incorporated into the flow of the practice and complement and extend (rather than replace) the efforts of the primary care team.

\section{References}

1. Wagner EH, Austin BT, Von Korff M. Organizing care for patients with chronic illness. Milbank Q 1996;74:511-44.

2. Bodenheimer T, Wagner EH, Grumbach K. Improving primary care for patients with chronic illness. JAMA 2002;288:1775-9.

3. Bodenheimer T, Wagner EH, Grumbach K. Improving primary care for patients with chronic illness: the Chronic Care Model. Part 2. JAMA 2002; 288:1909-14.

4. Grundy P, Hagan KR, Hansen JC, Grumbach K. The multi-stakeholder movement for primary care renewal and reform. Health Aff (Millwood) 2010;29: 791-8.

5. Bodenheimer T, Pham HH. Primary care: current problems and proposed solutions. Health Aff (Millwood) 2010;29:799-805.

6. Reid RJ, Coleman K, Johnson EA, et al. The group health medical home at year two: cost savings, higher patient satisfaction, and less burnout for providers. Health Aff (Millwood) 2011;29:835-43.

7. Rosenthal TC. The medical home: growing evidence to support a new approach to primary care. J Am Board Fam Med 2008;21:427-40.

8. U.S. Preventive Services Task Force. Recommendations. Available from: http://www.uspreventiveservicestaskforce. org/recommendations. Accessed December 27, 2011.

9. Whitlock EP, Polen MR, Green CA, Orleans T, Klein J; U.S. Preventive Services Task Force. Behavioral counseling interventions in primary care to reduce risky/harmful alcohol use by adults: a summary of the evidence for the U.S. Preventive Services Task Force. Ann Intern Med 2004;140:557-68.

10. Whitlock EP, Orleans CT, Pender N, Allan J. Evaluating primary care behavioral counseling interventions: an evidence-based approach. Am J Prev Med 2002;22:267-84.

11. Glasgow RE, Toobert DJ. Brief, computer-assisted diabetes dietary self-management counseling: effects on behavior, physiologic outcomes, and quality of life. Med Care 2000;38:1062-73.

12. Smith AW, Borowski LA, Liu B, et al. U.S. primary care physicians' diet-, physical activity-, and weightrelated care of adult patients. Am J Prev Med 2011; 41:33-42.

13. Stange KC, Woolf SH, Gjeltema K. One minute for prevention: the power of leveraging to fulfill the promise of health behavior counseling. Am J Prev Med 2002;22:320-3.

14. Yarnall KS, Pollak KI, Ostbye T, Krause KM, Michener JL. Primary care: is there enough time for prevention? Am J Public Health 2003;93:635-41.
15. Glasgow RE, Bull SS, Piette JD, Steiner JF. Interactive behavior change technology. A partial solution to the competing demands of primary care. Am J Prev Med 2004;27(2 Suppl):80-7.

16. Williams GC, Deci EL. Activating patients for smoking cessation through physician autonomy support. Med Care 2001;39:813-23.

17. Deci EL. On the nature and function of motivation theories. Psychol Sci 1992;3:168-71.

18. Williams GC, Lynch M, Glasgow RE. Computerassisted intervention improves patient-centered diabetes care by increasing autonomy support. Health Psychol 2007;26:728-34.

19. Williams GC, Niemiec CP, Ryan RM. The importance of supporting autonomy and perceived competence in facilitating long-term tobacco abstinence. Ann Behav Med 2009;37:315-24.

20. Wagner EH. Chronic disease management: what will it take to improve care for chronic illness? Eff Clin Pract 1998;1:2-4.

21. Estabrooks PA, Nelson CC, Xu S, et al. The frequency and behavioral outcomes of goal choices in the self-management of diabetes. Diabetes Educ 2005;31:391-400.

22. Estabrooks PA, Glasgow RE, Dzewaltowski DA. Physical activity promotion through primary care. JAMA 2003;289:2913-6.

23. Glasgow RE, Funnell MM, Bonomi AE, Davis C, Beckham V, Wagner EH. Self-management aspects of the improving chronic illness care breakthrough series: Implementation with diabetes and heart failure teams. Ann Behav Med 2002;24:80-7.

24. Tate DF, Jackvony EH, Wing RR. Effects of Internet behavioral counseling on weight loss in adults at risk for type 2 diabetes: a randomized trial. JAMA 2003;289:1833-6.

25. Glasgow RE, Christiansen S, Smith KS, Stevens VJ, Toobert DJ. Development and implementation of an integrated, multi-modality, user-centered interactive dietary change program. Health Educ Res 2008; 24:461-71.

26. Stevens VJ, Glasgow RE, Toobert DJ, Karanja N, Smith KS. One-year results from a brief, computerassisted intervention to decrease consumption of fat and increase consumption of fruits and vegetables. Prev Med 2003;36:594-600.

27. Glasgow RE, Kurz D, King D, et al. Outcomes of minimal versus moderate support versions of an Internet-based diabetes self-management support program. J Gen Intern Med 2010;25:1315-22.

28. Glasgow RE, Ory MG, Klesges LM, Cifuentes M, Fernald DH, Green LA. Practical and relevant self-report measures of patient health behaviors for primary care research. Ann Fam Med 2005;3: 73-81.

29. Fernald DH, Froshaug DB, Dickinson LM, et al. Common measures, better outcomes (COMBO): a field test of brief health behavior measures in pri- 
mary care. Am J Prev Med 2008;35(5 Suppl): S414-22.

30. Jilcott SB, Keyserling TC, Samuel-Hodge CD, Johnson LF, Gross MD, Ammerman AS. Validation of a brief dietary assessment to guide counseling for cardiovascular disease risk reduction in an underserved population. J Am Diet Assoc 2007; 107:246-55.

31. Paxton AE, Strycker LA, Toobert DJ, Ammerman AS, Glasgow RE. Starting the conversation: performance of a brief dietary assessment and intervention tool for health professionals. Am J Prev Med 2011; 40:67-71.

32. Craig CL, Marshall AL, Sjöström M, et al. International physical activity questionnaire: 12 -country reliability and validity. Med Sci Sports Exerc 2003;35: 1381-95.

33. Ory MG, Jordan PJ, Bazzare T. The Behavior Change Consortium: setting the stage for a new century of health behavior change research. Health Educ Res 2002;17:500-11.

34. Society for Research on Nicotine and Tobacco. Available from: http://hmcrc.srph.tamu.edu/Measurement\% 20folder/Smoking/Tobacco\%20SNT.pdf. Accessed 2013.

35. U.S. Department of Health and Human Services. Behavioral Risk Factor Surveillance System. 2004. Available from: http://www.cdc.gov/brfss/index.htm. Accessed January 5, 2013.

36. Kroenke K, Spitzer RL, Williams JB. The PHQ-9: validity of a brief depression severity measure. J Gen Intern Med 2001;16:606-13.
37. Moriarty DG, Kobau R, Zack MM, Zahran HS. Tracking healthy days-a window on the health of older adults. Prev Chronic Dis 2005;2:A16.

38. Littell RC, Milliken GA, Stroup WW, Wolfinger RD. SAS system for mixed models. Cary, NC: SAS Institute. Inc.; 1999.

39. Fairclough D. Design and Analysis of Quality of Life Studies in Clinical Trials. Boca Raton, FL: Chapman \& Hall/CRC.

40. Gibbons RD, Hedeker D, Elkin I, et al. Some conceptual and statistical issues in analysis of longitudinal psychiatric data: application to the NIMH treatment of depression collaborative research program dataset. Arch Gen Psychiatry 1993;50:739-50.

41. Brouwer $W$, Kroeze W, Crutzen R, et al. Which intervention characteristics are related to more exposure to Internet-delivered healthy lifestyle promotion interventions? A systematic review. J Med Internet Res 2011;13:e2.

42. Papadakis S, McDonald P, Mullen KA, Reid R, Skulsky K, Pipe A. Strategies to increase the delivery of smoking cessation treatments in primary care settings: a systematic review and meta-analysis. Prev Med 2010;51:199-213.

43. King DK, Estabrooks PA, Strycker L, Toobert D, Bull SS, Glasgow RE. Outcomes of a multifaceted physical activity regimen as part of a diabetes self-management intervention. Ann Behav Med 2006;31:128-37.

44. King DK, Glasgow RE, Toobert DJ, et al. Selfefficacy, problem solving, and social-environmental support are associated with diabetes self-management behaviors. Diabetes Care 2010;33:751-3. 